\title{
IL-18 knockout alleviates atopic dermatitis-like skin lesions induced by MC903 in a mouse model
}

\author{
JIA-LONG CHEN ${ }^{1-3}$, XUE-LI NIU ${ }^{1-3}$, YA-LI GAO ${ }^{1-3}$, LEI MA ${ }^{4}$, \\ XING-HUA GAO ${ }^{1-3}$, HONG-DUO $\mathrm{CHEN}^{1-3}$ and RUI-QUN QI ${ }^{1-3}$ \\ ${ }^{1}$ Graduate School, China Medical University; ${ }^{2}$ Department of Dermatology, The First Hospital of China Medical University; \\ ${ }^{3}$ Key Laboratory of Immunodermatology, Ministry of Health and Ministry of Education, Shenyang, Liaoning 110001; \\ ${ }^{4}$ Department of Dermatology, Binzhou Medical University Hospital, Binzhou, Shandong 256603, P.R. China
}

Received November 23, 2019; Accepted May 14, 2020

DOI: $10.3892 /$ ijmm.2020.4630

\begin{abstract}
Interleukin (IL)-18, a pro-inflammatory cytokine, plays an important role in a number of skin diseases. The aim of the present study was to investigate the role of IL-18 in the development of atopic dermatitis (AD). For this purpose, mice were divided into 4 groups ( $n=5 /$ group) as follows: i) The wild-type (WT) controls; ii) IL18 knockout (KO) controls; iii) MC903-treated WT mice; and iv) MC903-treated KO mice. MC903 (4 nmol in ethanol) was topically applied daily for 15 consecutive days to the exposed skins of mice. AD-like symptoms and severity were evaluated by the scoring of AD (SCORAD). Serum immunoglobulin E ( $\mathrm{IgE}$ ) and thymic stromal lymphopoietin (TSLP) levels were determined with the use of an enzyme-linked immunosorbent assay. Immunohistochemistry was used to assess the expression of IL-1 $\beta$, signal transducer and activator of transcription (STAT) 3 and filaggrin (FLG) in the skin lesions. RT-qPCR was performed to assess the mRNA levels of IL-1 $\beta$, IL-4, IL-9, STAT3, corticotropin-releasing hormone receptor (CRHR)1, CRHR2, TSLP and caspase-1 in the skin lesions. It was demonstrated that IL-18 may function as a pleiotropic pro-inflammatory cytokine in the development of AD-like lesions. IL-18 KO reduced aggravated AD-like lesions induced by MC903, in part by upregulating Th2 cytokines. IL-18 promoted the expression of FLG in the epidermis and CRHR2 in AD-like lesions, but downregulated the serum levels of $\mathrm{IgE}$.
\end{abstract}

Correspondence to: Professor Hong-Duo Chen, Department of Dermatology, The First Hospital of China Medical University, $155 N$ Nanjing Road, Heping, Shenyang, Liaoning 110001, P.R. China E-mail: hongduochen@hotmail.com

Dr Rui-Qun Qi, Key Laboratory of Immunodermatology, Ministry of Health and Ministry of Education, 155N Nanjing Road, Heping, Shenyang, Liaoning 110001, P.R. China

E-mail: xiaoqiliumin@163.com

Key words: interleukin-18, atopic dermatitis, cytokines, filaggrin, immunoglobulin, MC903
On the whole, the findings of the present study demonstrate that IL-18 deficiency alleviates AD-induced lesions in mice.

\section{Introduction}

Atopic dermatitis (AD) is the most common chronic inflammatory skin disease. This condition generally occurs in infancy and is characterized by pruritus, a chronically relapsing course of eczematous lesions and elevated serum IgE levels (1). AD is considered to be triggered by a complex combination of multiple factors. Risk factors for AD consist of environmental changes, genetic susceptibility, epidermal barrier abnormalities, the dysbiosis of skin microbiota and $\mathrm{T}$ cell-driven skin inflammation (2). All of the above-mentioned factors can lead to the clinical heterogeneity observed among patients with AD.

Interleukin (IL)-18 is generally expressed in AD. IL-18 respectively enhances Th1 and Th2 responses, including allergic inflammation under different surrounding cytokine environments (3). IL-18 was originally considered a Th1 cytokine through its ability to induce interferon (IFN)- $\gamma$ production (4). The activation of caspase-1 and cleaved pro-IL-1 $\beta$ and pro-IL18 result in the active forms of IL-1 $\beta$ and IL18, respectively. These active forms can then stimulate inflammatory responses (5). IL-18 has been reported to induce the production of $\operatorname{IgE}$ and Th2 cytokines, such as IL-4 and IL-13 (6). IL-18 has also been shown to be associated with the severity of $\mathrm{AD}(7)$. In addition, the scoring of AD (SCORAD), a recommended outcome assessment for the symptoms of $\mathrm{AD}$, has been shown to be positively associated with serum IL-18 levels in patients with AD $(8,9)$. However, the biological activities of IL-18, associate with a number of skin diseases, are not yet fully understood.

Thymic stromal lymphopoietin (TSLP) is a cytokine produced by keratinocytes, fibroblasts and mast cells upon exposure to allergens, viral infections, trauma and other cytokines. It is highly expressed in acute and chronic lesions of $\mathrm{AD}$ and is considered the 'master-switch of allergic inflammation' due to its central role in evoking Th2 responses via dendritic cell activation $(10,11)$. In general, AD is a complex, chronic inflammatory disease associated with environmental influences, underlying defects in the epidermal barrier and cytokine dysregulation. 
The pathogenic role of IL-18, as regards AD, remains to be determined. One approach to examine this association involves the use of animal models of AD. Such a model can be induced in mice through the topic application of calcipotriol (MC903), a low-calcemic analog of vitamin D3 $(12,13)$. In the present study, the effects of exposure to MC903 were examined in IL-18 knockout (KO) mice in order to investigate the effects of IL-18 on the development of AD-like lesions.

\section{Materials and methods}

Mice and treatment. Female wild-type (WT) and IL-18 KO C57BL/6 mice (12-15 weeks old, weight range, 22-25 g; $\mathrm{n}=20$ ) were purchased from Jackson Laboratory (stock no. C57BL/6:000664-JAX; IL-18 KO: 004130-JAX). Female mice were used as in general, female mice are considered less likely to exhibit aggressive behavior, which can lead to unnecessary skin injuries, which may have impeded the research purposes of the present study. According to Moosbrugger-Martinz et al (12), AD-like symptoms induced by MC903 were not dependent on mouse sex or on genetic background. An AD-like mouse model established using female mice is widely used by a number of researchers (13-15). In the present study, all mice were housed individually in ventilated cages under specific pathogen-free conditions at $22 \pm 2^{\circ} \mathrm{C}$ with a 12 -h light/dark cycle. All experimental protocols were strictly approved by the guide for the care and use of laboratory animals (NIH Publication, 8th Edition, 2011) (16) and were conducted according to the guidelines provided and approved by the Institutional Animal Care and Use Committee at China Medical University (IACUC no. 16008M). The mice were divided into 4 groups (n=5/group) as follows: i) The WT control; ii) IL-18 KO control; iii) MC903-treated WT mice; and iv) MC903-treated IL-18 KO mice.

To establish an AD-like mouse model, all mice were shaved on their dorsal skin surface to expose a $2 \times 3 \mathrm{~cm}$ area. MC903 (4 nmol; Sigma-Aldrich; Merck KGaA) diluted in ethanol was applied once a day topically to the exposed skin for 15 consecutive days according to previously published protocols $(12,14,17)$. The mice in the control groups received an identical volume of ethanol. Skin samples were collected from all mice on day 16.

Scoring the severity of skin inflammation. To evaluate the severity of AD, the scoring of dermatitis was conducted in each group on days $0,1,3,5,7,9,11,13$ and 15 . The scoring of dermatitis (SCORAD) represents an established method with which to assess the signs of $\mathrm{AD}(9,18)$. This method consists of scoring the following factors on a scale from 0-3: Erythema, papule/edema, exudation/crust, excoriation, fissures and lichenification. The scores were classified as follows: 0 (none), 1 (mild), 2 (moderate) and 3 (severe) and were recorded independently by 2 investigators on every two 2 prior to the daily application of MC903.

Sample collection. The mice were anesthetized by 3-4\% isoflurane for the induction and $1.5-2 \%$ for the maintenance of anesthesia to finish daily application of MC903. On day 16, all mice were euthanized by raising to $10 \%$ isoflurane for 5 min following the induction of anesthesia. Then they were transferred to a closed container with significantly high dose isoflurane for sample collection. When the mice were fully sedated (by the lack of active paw reflex, and the termination of breathing for $>2 \mathrm{~min}$ ), blood samples were then obtained by cardiac puncture. Serum was collected after centrifugation and stored at $-80^{\circ} \mathrm{C}$. Treated skin areas were obtained and then divided into 2 sections. One section was stored at $-80^{\circ} \mathrm{C}$ immediately for the extraction of total RNA and the second was fixed with $4 \%$ paraformaldehyde for histopathological analysis and immunohistochemistry.

Histopathological examination. The collected tissues fixed with $4 \%$ paraformaldehyde were embedded in paraffin and cut into $5-\mu \mathrm{m}$-thick sections. Deparaffinized tissue sections were stained with hematoxylin and eosin (Solarbio, G1121) and Toluidine blue (Solarbio, G3663) using standard procedures at room temperature. The staining duration for staining with hematoxylin, eosin and toluidine blue was 5, 1 and $10 \mathrm{~min}$, respectively. All sections were examined by 2 independent pathologists with use of an Olympus CHB213 light microscope (Olympus Corporation) at $\mathrm{x} 400$ magnification to measure the thickness of the epidermis and to count the number of neutrophils, eosinophils, histocytes and mast cells per $0.025 \mathrm{~mm}^{2}$ of dermis. The distance from the stratum corneum to the basement membrane zone were measured as the thickness of the epidermis. The average maximum thickness at 3 high magnification fields was obtained by 2 independent individuals. The number of neutrophils, eosinophils, histocytes and mast cells per $0.025 \mathrm{~mm}^{2}$ of dermis were counted.

Immunohistochemistry. Tissue sections were incubated overnight at $4^{\circ} \mathrm{C}$ with IL-1b polyclonal rabbit antibody (dilution, 1:100; cat. no. ab9722, Abcam), mouse Anti-filaggrin (FLG) rabbit polyclonal antibody (dilution, 1:100; cat. no. TA323075, Origene) or signal transducer and activator of transcription (STAT)3 rabbit monoclonal antibody (dilution, 1:300; cat. no. ab31370, Abcam). The EliVision Super Kit (Maixin) was then used for immunostaining. A total of 5 views were selected in the center of each tissue slide for evaluation. The mean integral optic density (MIOD) was used to analyze overall protein expression as calculated by dividing the integral optic density (IOD) value by area obtained with the use of Image-Pro Plus 6.0 software (Media Cybernetics, Inc.).

Determination of total serum IgE levels and TSLP. Serum levels of total IgE and TSLP were measured with the use of mouse serum enzyme-linked immunosorbent assay kits (Thermo Fisher Scientific, Inc. and R\&D Systems, Inc.) according to the manufacturers' protocols.

RNA isolation and reverse transcription-quantitative PCR. Total RNA was extracted from the treated skin of each mouse with the use of an miRNeasy mini kit (Qiagen $\mathrm{GmbH}$ ) according to the manufacturers' instructions and quantified with a ND1000 Nanometer. Complementary DNA (cDNA) was synthesized by utilizing $1 \mu \mathrm{g}$ mRNA extracted as templates with the GoScript Reverse Transcription kit (Promega Corp.) according to the protocols supplied. qPCR was performed in 384-well plates with use of RT2 SYBR-Green qPCR Mastermix (Promega Corp.) and the 7900 HT Fast Real-Time PCR system (Applied Biosystems). The real-time qPCR mix 
A
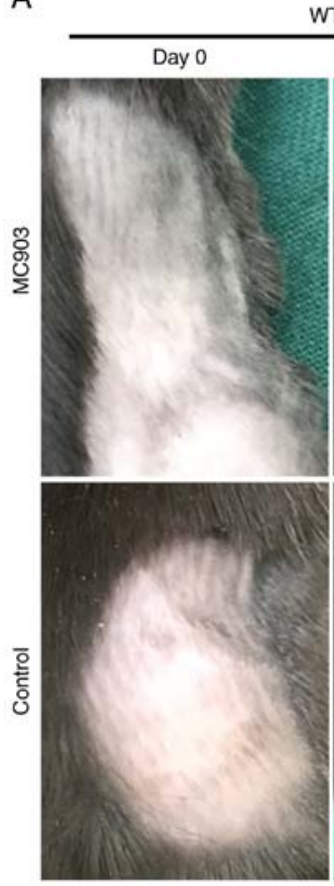

B

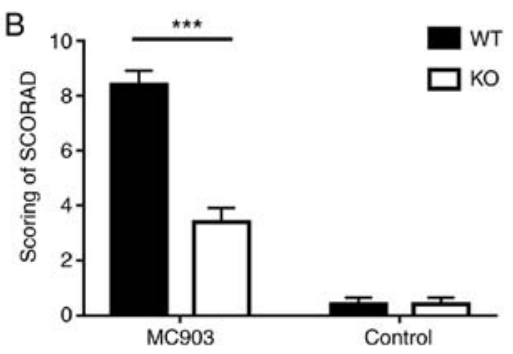

WT

Day 16
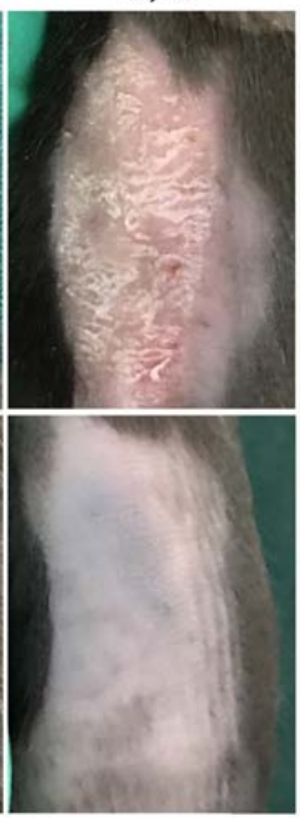

KO

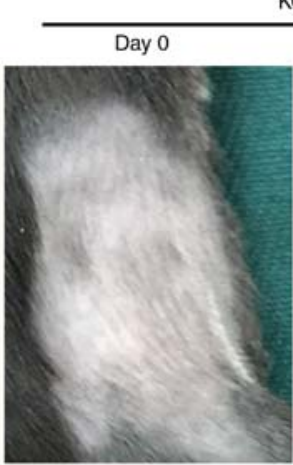

Day 16
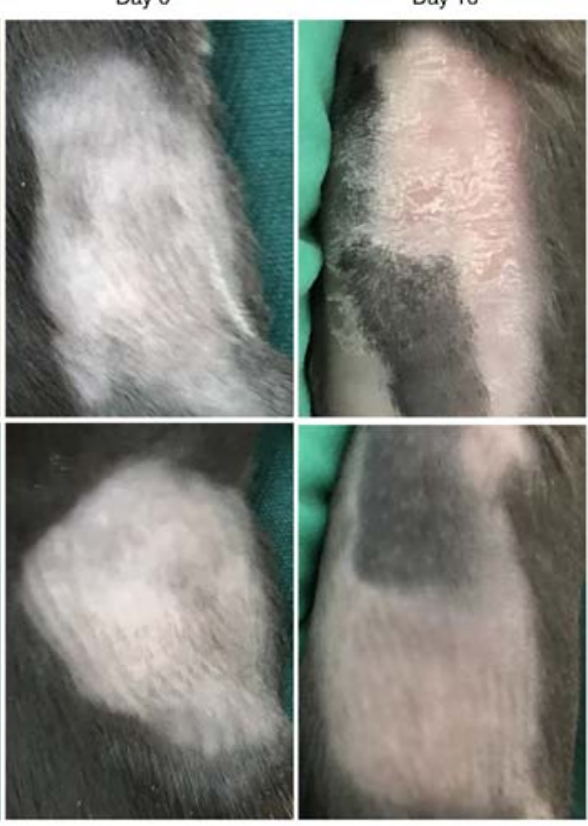

C

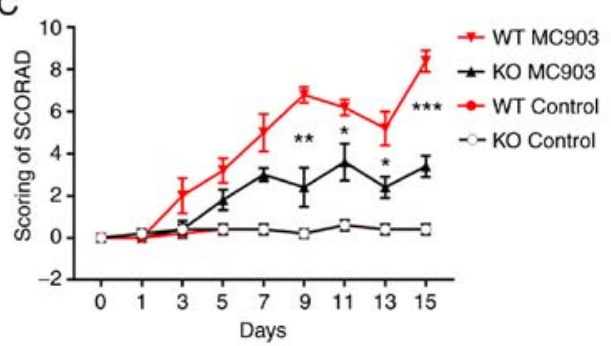

Figure 1. (A) Topical application of MC903 induces AD-like lesions in WT and KO mice, (B) Scoring of dermatitis (SCORAD) revealed higher scores in WT mice vs. KO mice treated with MC903 (WT mice, $8.4 \pm 0.5099$; KO mice, $3.4 \pm 0.5099$, ${ }^{* * *} \mathrm{P}=0.0001$ ). No statistically significant differences were observed between WT mice and KO controls (WT mice, $1.8 \pm 0.3742 ; \mathrm{KO}$ mice, $1.4 \pm 0.2449, \mathrm{P}>0.05$ ). Data are expressed as the means \pm SEM. (C) SCORAD was conducted on days $0,1,3,5,7,9,11,13$ and 15 following MC903 treatment. ${ }^{*} \mathrm{P}<0.05,{ }^{* *} \mathrm{P}<0.01,{ }^{* * *} \mathrm{P}<0.001$. AD, atopic dermatitis; WT, wild-type; KO, IL-18 knockout.

contained $0.2 \mu 1$ of each primer, $0.1 \mu \mathrm{l}$ CXR Reference Dye, $5 \mu 1$ qPCR Master Mix and $1 \mu 1$ of cDNA. Nuclease-free water was added to achieve a final reaction volume of $10 \mu 1$. The real-time PCR reaction conditions were set to $95^{\circ} \mathrm{C}$ for 2 min, followed by 40 cycles at $95^{\circ} \mathrm{C}$ prolongation for $15 \mathrm{sec}$ and $60^{\circ} \mathrm{C}$ for $1 \mathrm{~min}$ each. A melting curve was then calculated for each PCR product to confirm synthesis specificity. The Ct value was calculated with use of the RQ Manager software (Applied Biosystems). Glyceraldehyde 3-phosphate dehydrogenase (GAPDH) was used as the internal control and expression levels of target genes were calculated by applying the $2^{-\Delta \Delta C \mathrm{Cq}}$ method (19). The experiments were repeated 3 times. The primer sequences for GAPDH IL-1 $\beta$, IL-4, IL-9, STAT3, corticotropin-releasing hormone receptor (CRHR2) TSLP and caspase-1 were designed using Primer-Premier 6.0 software. The sequences of the primers were as follows: GAPDH sense, 5'-GTGCTCTCTGCTCCTCCCTGT-3' and antisense, 5'-CGGCCAAATCCGTTCACACCG-3'; IL-1 $\beta$ sense, 5'-CAC TACAGGCTCCGAGATGAA-3' and antisense, 5'-TGTCGT TGCTTGGTTCTCCT-3'; IL-4 sense, 5'-GAACTCTAGTGT TCTCATGGAGC-3' and antisense, 5'-AGTGATGTGGAC TTGGACTCAT-3'; IL-9 sense, 5'-CAACCTGCTGACATT CTACAGAG-3' and antisense, 5'-CCTGACATCGCTCCA
GAGATTT-3'; STAT3 sense, 5'-AGGACATCAGTGGCA AGACC-3' and antisense, 5'-GTAGAGGTAGACAAGTGG AGACA-3'; CRHR2 sense, 5'-GACCACGGGAAGTGAG TTA-3' and antisense, 5'-TCCCAGGCATACTCTGATTT-3'; caspase-1 sense, 5'-CGTGGAGAGAAACAAGGAGTG-3' and antisense, 5'-TGGTGTTGAAGAGCAGAAAGC-3'.

Statistical analysis. Data are expressed as the mean and the standard error of mean (SEM) and statistically significant differences between 2 groups were determined using the Student's t-test. Results were considered statistically significant when $\mathrm{P}<0.05$. GraphPad Prism 5 (GraphPad Software, Inc.) was used for statistical analysis and for plotting the graphs.

\section{Results}

IL-18 is associated with the severity of MC903-induced AD-like lesions. An IL-18 deficiency alleviated the AD-like inflammatory symptoms induced by MC903 in mice. More severe degrees of erythema, edema, scaling, exudation and crusts were observed in the MC903-treated skin samples of the WT vs. the IL-18 KO mice. By contrast, all mice in the control groups exhibited mild erythema (Fig. 1A). The 
A

WT

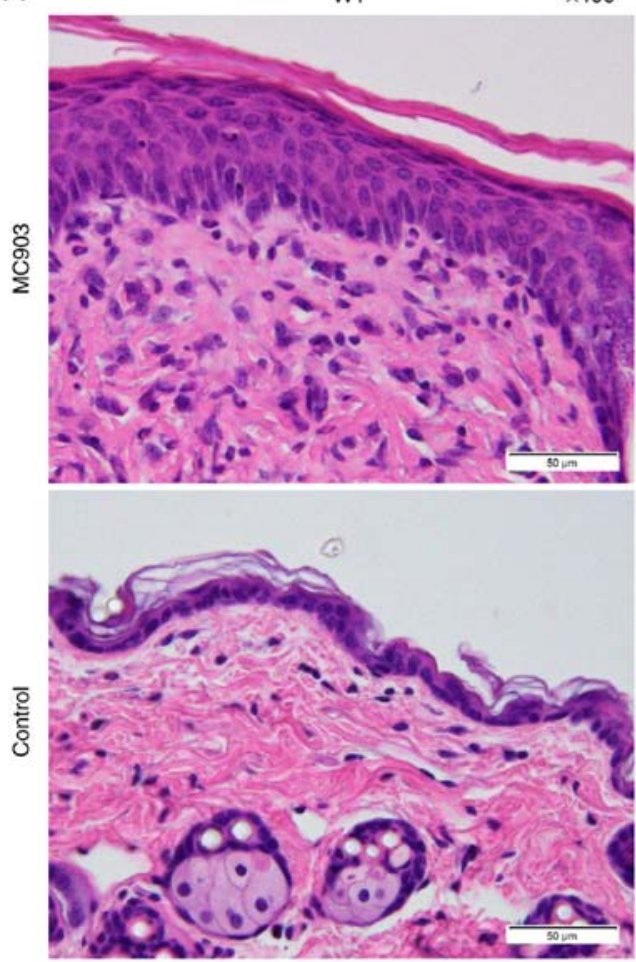

B

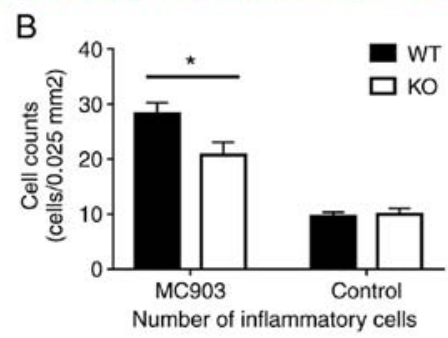

$\times 400$
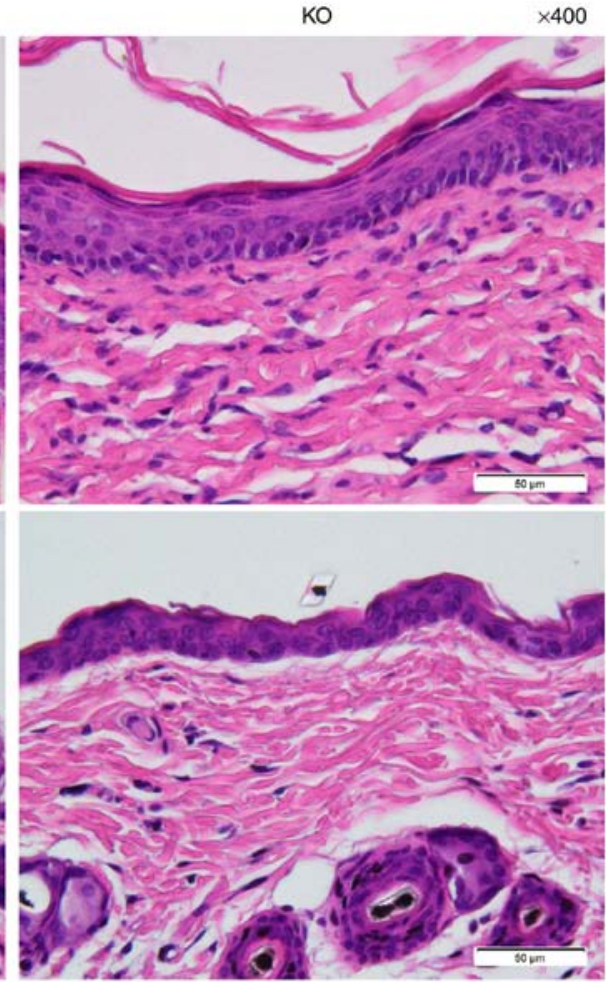

C

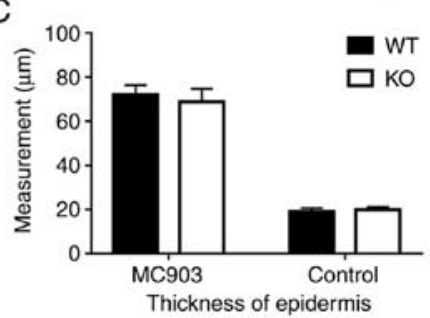

Figure 2. (A) Histopathological examination of treated skin as assessed by H\&E staining. MC903-treated skin exhibited clear evidence of inflammatory cell infiltration. (B) Counts of total inflammatory cells in the dermis. Data are expressed as the means \pm SEM of cells per 0.025 mm² (MC903: WT mice, $28.24 \pm 1.996$; KO mice, 20.8 $\pm 2.274,{ }^{*} \mathrm{P}<0.05$; controls: WT mice, 9.55 \pm 0.7915 ; KO mice, 5.9.95 $\left.\pm 1.141, \mathrm{P}>0.05\right)$. (C) Mean $\pm \mathrm{SEM}$ of epidermal thickness in $\mu \mathrm{m}$ (MC903: WT mice, 72.22 \pm 4.212 ; KO mice, 68.77 \pm 5.982 , P>0.05; controls: WT mice, 19.07 \pm 1.615 ; KO mice, 19.99 \pm 1.247 , P>0.05). WT, wild-type; KO, IL-18 knockout.

SCORAD values in the MC903-treated mice were significantly increased in the WT as compared with the IL-18 KO mice (WT mice, 8.4 $\pm 0.5099 ; \mathrm{KO}$ mice, 3.4 $\pm 0.5099 ; \mathrm{P}=0.0001$; Fig. 1B and C). These results are very similar with those reported previously (8).

IL-18 deficiency attenuates AD-like inflammation induced by MC903 in mice. Compared to the control groups, histopathological examination of the skin samples of the MC903-treated mice revealed acanthosis, hyperkeratosis and parakeratosis. An increased number of inflammatory cells infiltrated the dermis, including neutrophils, histocytes, eosinophils, mast cells and lymphocytes, along with dermal fibrosis. Lymphocytes occasionally extended into the epidermis (Fig. 2A). These results are consistent with those reported previously $(13,20)$. In the MC903-induced AD-like lesions, IL-18 deficiency resulted in lower numbers of total inflammatory cell infiltration than that observed in the WT mice (WT mice, 28.24 \pm 1.996 cells per $0.025 \mathrm{~mm}^{2}$; KO mice, $20.8 \pm 2.274$ cells per $0.025 \mathrm{~mm}^{2}$; $\mathrm{P}<0.05$; Fig. 2B).

However, IL-18 deficiency failed to affect epidermal thickness in the MC903-induced AD-like skin lesions (control:
WT mice, $19.07 \pm 1.615 \mu \mathrm{m}$ vs. KO mice, $19.99 \pm 1.247 \mu \mathrm{m}$, $\mathrm{P}>0.05$; MC903-treated mice: WT mice, $72.22 \pm 4.212 \mu \mathrm{m}$ vs. $\mathrm{KO}$ mice, 68.77 $\pm 5.982 \mu \mathrm{m}, \mathrm{P}>0.05$; Fig. 2C).

Mast cells labeled with Toluidine blue were used as a means to assess MC903-induced AD-like lesions in the mouse model. Significant differences were found between the WT and KO mice in the AD-like lesions induced by MC903. Mast cell counts in the WT mice were significantly higher than those in the KO mice (WT mice, 9.76 \pm 0.6882 cells per $0.025 \mathrm{~mm}^{2}$; KO mice, $6.68 \pm 0.989$ cells per $0.025 \mathrm{~mm}^{2} ; \mathrm{P}<0.05$; Fig. 3A and B). No statistically significant differences were observed between the number of eosinophils in the WT vs. KO AD-like lesions (WT mice, $1.04 \pm 0.2926$ cells per $0.025 \mathrm{~mm}^{2}$; $\mathrm{KO}$ mice, $1.28 \pm 0.3072$ cells per $0.025 \mathrm{~mm}^{2}$; P>0.05; Fig. $3 \mathrm{C}$ ).

IL-18 deficiency exerts no effects on TSLP levels, but upregulates serum IgE levels. The serum levels of TSLP and total IgE within both WT and IL-18 KO mice were markedly increased in response to MC903 treatment. However, no significant differences were observed for the serum levels of TSLP between the WT and IL-18 KO mice (MC903 group: WT mice, $192 \pm 50.29 \mathrm{pg} / \mathrm{ml}$; KO mice, $128.3 \pm 13.94 \mathrm{pg} / \mathrm{ml}$, 
A

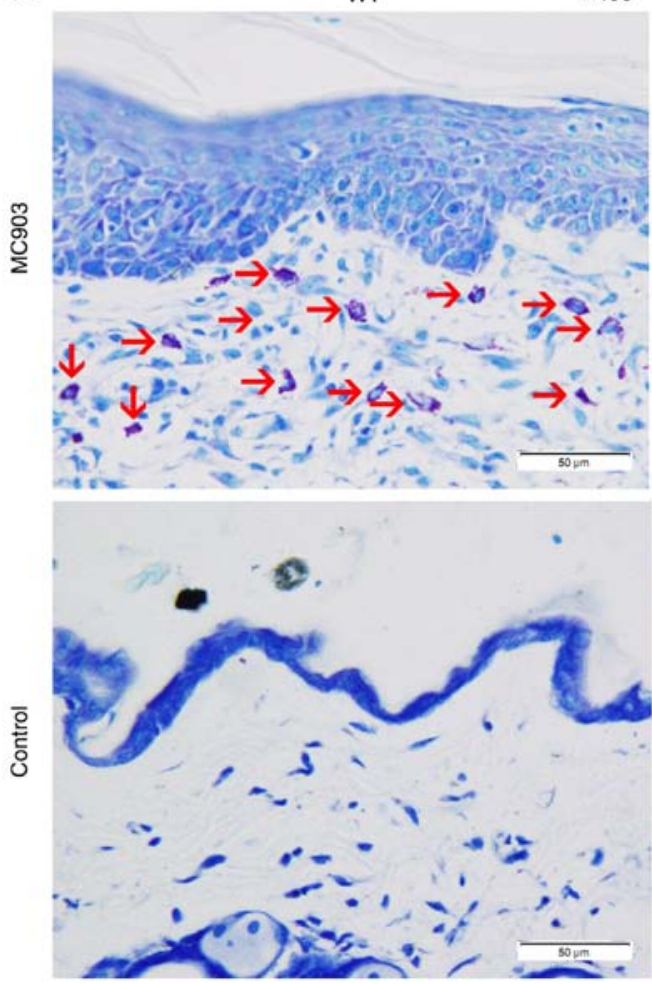

B

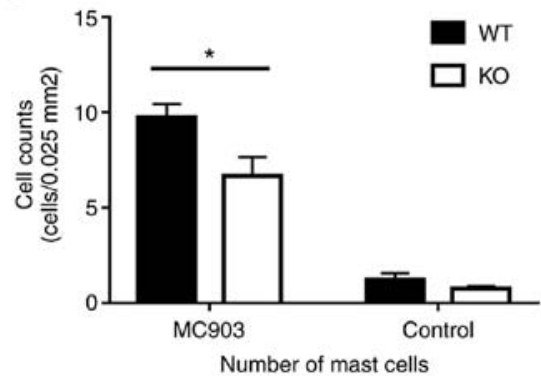

KO
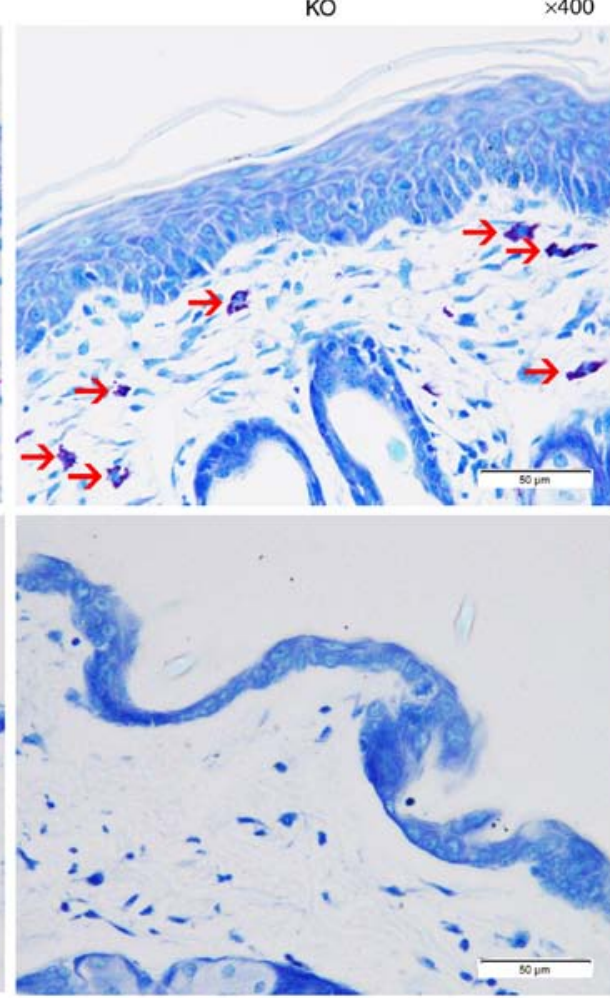

C

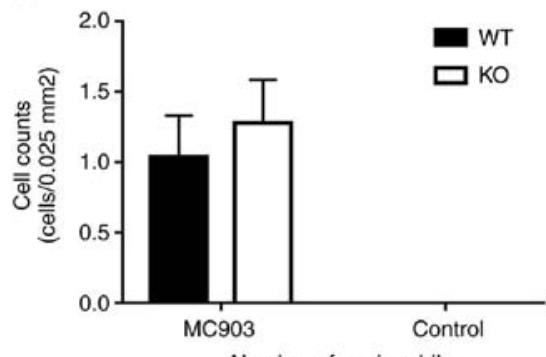

Figure 3. (A) Mast cells stained with Toluidine blue within the dermis of each group (mast cells are indicated by red arrows). (B) Counts of mast cells in the dermis; data are expressed as the means \pm SEM, cells per $0.025 \mathrm{~mm}^{2}$ (MC903: WT mice, 9.76 \pm 0.6882 ; KO mice, $6.68 \pm 0.989$, ${ }^{*}<<0.05$; controls: WT mice, 1.24 \pm 0.337 ; KO mice, $0.76 \pm 0.1166$, P>0.05). (C) Counts of eosinophils in the dermis of MC903 treated WT and KO mice; data are expressed as the means \pm SEM, cells per $0.025 \mathrm{~mm}^{2}$ (WT mice, $1.04 \pm 0.2926$; KO mice, $1.28 \pm 0.3072$, P $>0.05$ ). No eosinophils were observed in the skin samples of the controls. WT, wild-type; KO, IL-18 knockout.

P>0.05; control group: WT mice, $5.879 \pm 0.47 \mathrm{pg} / \mathrm{ml}$; KO mice, $6.504 \pm 0.68 \mathrm{pg} / \mathrm{ml}, \mathrm{P}>0.05$; Fig. $4 \mathrm{~A})$. As such, the mRNA levels of TSLP within the skin tissue also exhibited no significant differences ( $\mathrm{P}>0.05$, Fig. 4C). However, the serum levels of total $\mathrm{IgE}$ in the WT mice were significantly decreased as compared with those in the IL-18 KO mice for both the MC903-treated and control mice (MC903 group: WT mice, 518.4 \pm 42.82 ; KO mice, 637.4 $\pm 28.22, \mathrm{P}<0.05$; control group: WT mice, 41.07 \pm 3.856 ; KO mice, 59.21 $\pm 4.666, \mathrm{P}<0.05$; Fig. 4B).

IL-18 partially upregulates the mRNA levels of proinflammatory Th2 type cytokines in MC903-induced AD-like lesions. Considering the crucial role of inflammatory cytokines in the development of AD, the expression levels of IL-1 $\beta$, IL-4, IL-9, STAT3, TSLP and caspase-1 in the skin lesions were assessed. As shown in Fig. 6, in both the MC903-treated and control IL-18 KO mice, the relative mRNA expression levels of IL-1 $\beta$, IL-9, STAT3 and caspase- 1 in the WT mice did not differ significantly ( $\mathrm{P}>0.05$, Fig. 6). Similar results were obtained by immunohistochemistry for IL-1 $\beta$ and STAT3 (Fig. 5). However, IL-18 significantly upregulated the mRNA expression levels of IL-4 and CRHR2 in the MC903-induced AD-like lesions, as shown in the MC903-treated WT mice $(\mathrm{P}<0.05$ and $\mathrm{P}<0.01$, respectively, Fig. 6), whereas the mRNA expression of CRHR1 exhibited no statistically significant difference $(\mathrm{P}>0.05$, data not shown).

IL-18 upregulates the expression of FLG in the epidermis of MC903-induced AD-like lesions. FLG is a keratin filament-aggregating protein that serves as a major structural component of the stratum corneum. When FLG is broken down, its products such as histidine contribute to epidermal hydration, acid mantle formation, lipid processing, and barrier function $(21,22)$. In the present study, the results from immunohistochemistry revealed that a lower expression of FLG was 

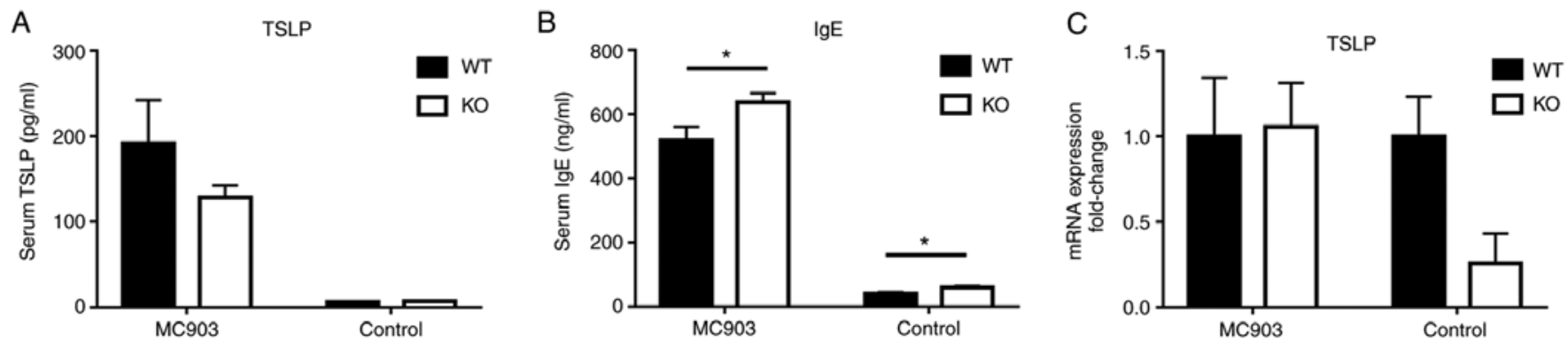

Figure 4. (A) Serum levels of TSLP; data are expressed as the means \pm SEM, pg/ml (MC903: WT mice, 192 \pm 50.29 ; KO mice, 128.3 $\pm 13.94, P>0.05$; controls: WT mice, 5.879 \pm 0.47 ; KO mice, $6.504 \pm 0.68, \mathrm{P}>0.05$ ). (B) Serum levels of total IgE; data are expressed as the means \pm SEM, ng/ml. (MC903: WT mice, 518.4 \pm 42.82 ; KO mice, $637.4 \pm 28.22$, ${ }^{*} \mathrm{P}<0.05$; controls: WT mice, 41.07 \pm 3.856 ; KO mice, $\left.59.21 \pm 4.666,{ }^{*} \mathrm{P}<0.05\right)$. (C) No statistically significant differences were observed in the skin tissue mRNA expression of TSLP were obtained between WT and KO mice (P>0.05). WT, wild-type; KO, IL-18 knockout; TSLP, thymic stromal lymphopoietin.

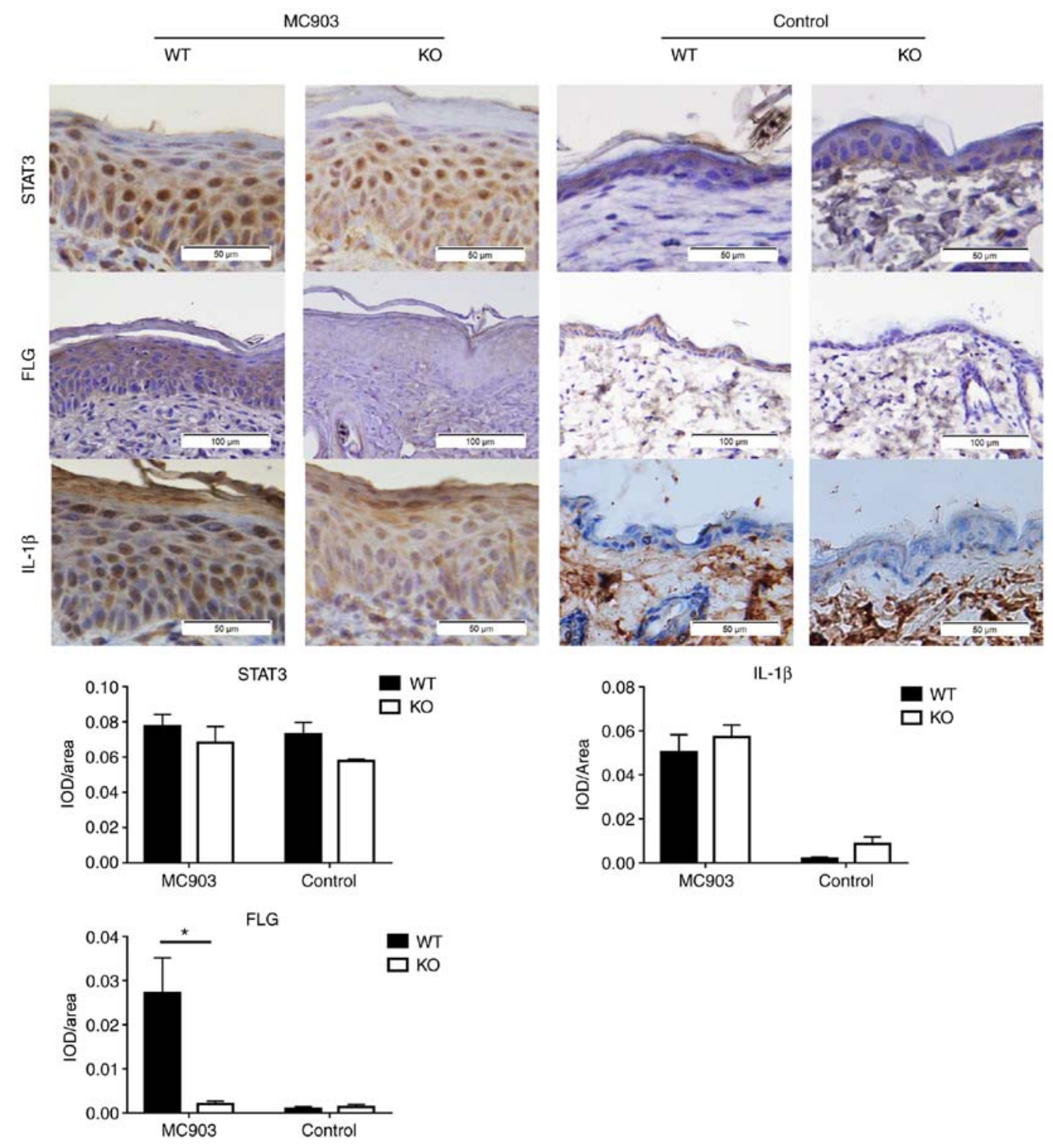

Figure 5. Immunohistochemistry of IL-1 $\beta$, FLG and STAT3 in induced AD-like lesioned skin samples. In both MC903-treated and control groups, the expression levels of IL-1 $\beta$ and STAT3 exhibited no statistically significant differences between the WT and KO mice (P>0.05). However, the expression of FLG in the KO mice following MC903 treatment was significantly decreased as compared with that in WT mice ( $\mathrm{P}<0.05)$, while no differences were obtained between WT and KO controls (P>0.05). WT, wild-type; KO, IL-18 knockout; IL, interleukin; FLG, filaggrin; STAT3, signal transducer and activator of transcription 3.

present in the epidermis of the MC903-treated IL-18 KO mice vs. the MC903-treated WT mice $(\mathrm{P}<0.01$; Fig. 5). By contrast, no differences were observed in FLG expression between the WT and IL-18 KO control mice (P>0.05; Fig. 5). 

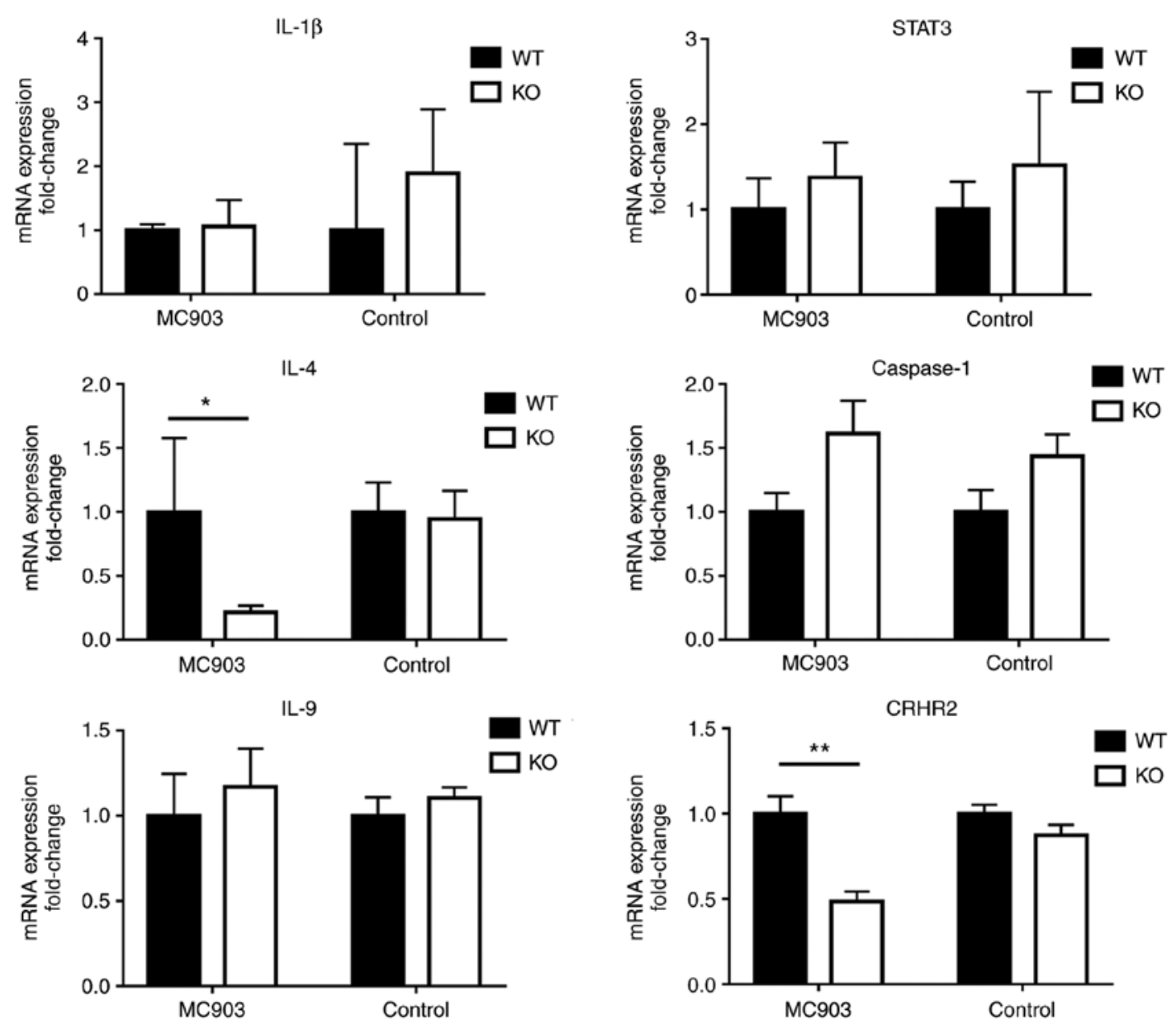

Figure 6. No statistically significant differences were found between WT and KO control or MC903 treated mice were obtained for IL-1 $\beta$, IL-9, STAT3 and caspase-1 mRNA expression $(\mathrm{P}>0.05)$. In response to MC903 treatment, IL-4 and CRHR2 mRNA expression in WT mice was significantly upregulated as compared with that observed in $\mathrm{KO}$ mice $\left({ }^{*} \mathrm{P}<0.05\right.$ and ${ }^{* *} \mathrm{P}<0.01$, respectively), while no statistically significant differences were obtained between the WT and KO controls (P>0.05). WT, wild-type; KO, IL-18 knockout; IL, interleukin; CRHR2, corticotropin-releasing hormone receptor 2; STAT3, signal transducer and activator of transcription 3

\section{Discussion}

In the present study, IL-18 KO reduced the production of pro-inflammatory cytokines and chemokines, and the infiltration of mast cells in AD. An imbalance between Th1 and $\mathrm{Th} 2$ immune functions has been proven to be a critical factor in the development of AD. Th2 cytokines are known to signal through JAK-dependent pathways in immune cells (23). The Th2 cytokines, IL-4 and IL-13, are essential for disease pathogenesis and the eventual development of the chronic itch associated with $\mathrm{AD}$, as sensory neurons are directly activated by IL-4 and IL-13 along itch-sensory pathways in mice (24). Activated STAT3 and STAT5 are required for mast cell function, which then participate in the pathogenesis of AD by producing pro-inflammatory cytokines and chemokines (25). The present study demonstrated that IL-18 KO reduced mRNA expression of IL-4 in AD-like lesions (Fig. 6). Moreover, in the mouse model of MC903-induced AD-like skin lesions, it was found that the numbers of mast cells in the WT mice were significantly increased as compared with the KO mice (Fig. 3A and B). Thus, degranulation is decreased and lower amounts of chemotaxis resulting from mast cells appear to contribute to an alleviation in the development of AD-like lesions.
Generally, IL-18 is involved in the phosphorylation of STAT3 and promotes cell proliferation $(26,27)$. However, the present study demonstrated IL-18 KO did not affect STAT3 expression in the process of AD-like lesions. STAT3 is required for Th2 cytokine production, transcription factor expression and $\mathrm{Th} 2$ cell-mediated allergic inflammation. In the presence of activated STAT6, STAT3 promotes Th2 cell differentiation and cytokine production (28). Serum levels of STAT3 have been shown to be increased in childhood AD and exhibit a significant positive association with SCORAD indices (29). STAT3 also plays an important role in the proximal signaling involved in IgE-dependent mast cell degranulation (30). In the present study, the expression of STAT3 exhibited no significant differences between the WT and KO mice during the development of AD-like lesions (Fig. 6). By contrast, the serum IgE levels were significantly increased and differed significantly between the WT and KO mice in this mouse model of AD (Fig. 4B). As a result, the capacity for Th2 induction of the JAK-STAT3 pathway was partially affected by IL-18 deficiency during the process of the development of AD-like lesions.

IL-18 KO may alleviate the inflammation of AD like lesions by reducing CRHR2 expression. Acute stress leads to increased skin vascular permeability and inflammation, through mast cell activation by CRH $(31,32)$. CRHR1 
and CRHR2 have been identified within the skin, and are expressed by epidermal keratinocytes, mast cells, melanocytes, peripheral lymphocytes and dermal blood vessel endothelial cells (33). CRH increases vascular permeability through the degranulation of mast cells via CRHR1 receptors (34) and can also directly lead to vasodilation mediated by CRHR2 receptors via a nitric oxide-cGMP-dependent pathway (35). A previous study (36) indicated lower levels of CRHR1 gene expression within the skin of AD patients as compared with the controls, while serum CRH levels in patients with AD were higher than those in the controls. In the present study, however, the mouse model of MC903-induced AD-like lesions exhibited a lower expression of CRHR2 (Fig. 6), as well as decreased amounts of mast cell infiltration (Fig. 3A and B). In the absence of IL-18, the expression of CRHR1 exhibited no marked differences in the skin samples from WT vs. KO mice (data not shown).

IL-18 may exert a negative effect on serum IgE levels and acts as a protective factor to the function of FLG. A previous study revealed that the knockdown of FLG increased the production of IL-18 in stratified human keratinocytes (37). It was uncertain however, whether FLG dysfunction affected the outcome of the AD process. FLG dysfunction mutations represent the most well-known genetic risk factor for AD. FLG expression is affected by intragenic copy number variation and reduced by increased local $\mathrm{pH}$, protease activity, and Th2 cytokine levels (1). Of note, IgE represents an established factor associated with autoantibodies and disease severity $(38,39)$ and patients with lower serum IgE levels exhibit a significantly lower incidence of FLG mutations and a higher percentage of IFN $\gamma$-producing Th1 cells (40). In the present study, a higher expression of FLG was found in the epidermis of MC903-treated WT mice vs. MC903-treated KO mice (Fig. 5). As noted previously, serum IgE levels of WT mice were significantly decreased as compared to KO mice (Fig. 4B). It seems likely that IL-18 may act as a protective factor in epidermal barrier dysfunction resulting from AD lesions.

In conclusion, during the process of AD-like lesion development, IL-18 deficiency partially alleviates Th2-induced inflammation by reducing the expression of the Th2 cytokine, IL-4. In addition, an IL-18 deficiency also reduces the infiltration of mast cells. On the other hand, IL-18 helped protect the epidermal barrier under normal FLG and lower serum IgE. As a result, in IL-18 KO mice, MC903-induced AD lesions exhibited a partial improvement as regards skin inflammation. Thus, the present study revealed that IL-18 may function as a pleiotropic cytokine and may be beneficial factor in AD development.

The mechanisms of action of AD however, warrant further in-depth investigations. A limitation of the present study is that it did not determine whether IL-18 plays a key role in the interaction between the serum levels of IgE and FLG mutation. Moreover, IL-18 KO seemed to exert a significant advantage by improving $\mathrm{AD}$-like lesions.

It is true that the phosphorylation of STAT3 in IL-18 KO mice was inhibited (41). In addition, caspase- 1 has cleaved and inactive forms, and they cannot be distinguished by RT-qPCR. The measurement of the phosphorylation of STAT3 and caspase- 1 is necessary for the investigation of the mechanisms of IL-18 in the pathogenesis of AD. The authors aim to perform further studies in the future to more deeply investigate the functions of IL-8, and the associated phosphorylation of STAT3 and caspase-1 in AD-like skin lesions.

\section{Acknowledgements}

Not applicable.

\section{Funding}

The present study was supported by the National Natural Science Foundation of China (grant nos. 81673070 and 81872538).

\section{Availability of data and materials}

All data generated or analyzed during this study are included in this published article or are available from the corresponding author on reasonable request.

\section{Authors' contributions}

JLC and RQQ made substantial contributions to the conception and design of the study. JLC performed the model established, histological examination of the skin, and was a major contributor in writing the manuscript. XLN and YLG performed the RT-qPCR analysis. LM was involved in data acquisition, analysis and interpretation. HDC and XHG were involved in the conception and design of this study. All authors read and approved the final manuscript and agree to be accountable for all aspects of the work in ensuring that questions related to the accuracy or integrity of the work are appropriately investigated and resolved.

\section{Ethics approval and consent to participate}

All experimental protocols were strictly approved by the guide for the care and use of laboratory animals (NIH Publication, 8th Edition, 2011) and were conducted according to the guidelines provided by the Institutional Animal Care and Use Committee at China Medical University (IACUC no. $16008 \mathrm{M})$.

\section{Patient consent for publication}

Not applicable.

\section{Competing interests}

The authors declare that they have no competing interests.

\section{References}

1. Bolognia J, Schaffer J and Cerroni L: Dermatology. 4th edition. Elsevier, Ltd., 2018.

2. Weidinger S, Beck LA, Bieber T, Kabashima K and Irvine AD: Atopic dermatitis. Nat Rev Dis Primers 4: 1, 2018.

3. Lee JH, Cho DH and Park HJ: IL-18 and cutaneous inflammatory diseases. Int J Mol Sci 16: 29357-29369, 2015.

4. Okamura H, Tsutsi H, Komatsu T, Yutsudo M, Hakura A, Tanimoto T, Torigoe K, Okura T, Nukada Y, Hattori K, et al: Cloning of a new cytokine that induces IFN-gamma production by T cells. Nature 378: 88-91, 1995. 
5. Sá DC and Festa CN: Inflammasomes and dermatology. An Bras Dermatol 91: 566-578, 2016.

6. Wild JS, Sigounas A, Sur N, Siddiqui MS, Alam R, Kurimoto M and Sur S: IFN-gamma-inducing factor (IL-18) increases allergic sensitization, serum IgE, Th2 cytokines, and airway eosinophilia in a mouse model of allergic asthma. J Immunol 164: 2701-2710, 2000.

7. Hon KL, Tsang KY, Kung JS, Leung TF, Lam CW and Wong CK: Clinical signs, staphylococcus and atopic eczema-related seromarkers. Molecules 22: 291, 2017.

8. Zedan K, Rasheed Z, Farouk Y, Alzolibani AA, Bin Saif G, Ismail HA and Al Robaee AA: Immunoglobulin E, interleukin-18 and interleukin-12 in patients with atopic dermatitis: Correlation with disease activity. J Clin Diagn Res 9: WC01-WC05, 2015.

9. Chopra R, Vakharia PP, Sacotte R, Patel N, Immaneni S, White T, Kantor R, Hsu DY and Silverberg JI: Severity strata for eczema area and severity index (EASI), modified EASI, scoring atopic dermatitis (SCORAD), objective SCORAD, atopic dermatitis severity index and body surface area in adolescents and adults with atopic dermatitis. Br J Dermatol 177: 1316-1321, 2017.

10. Ziegler SF and Artis D: Sensing the outside world: TSLP regulates barrier immunity. Nat Immunol 11: 289-293, 2010.

11. Eyerich $\mathrm{K}$ and Novak $\mathrm{N}$ : Immunology of atopic eczema: Overcoming the Th1/Th2 paradigm. Allergy 68: 974-982, 2013.

12. Moosbrugger-Martinz V, Schmuth M and Dubrac S: A mouse model for atopic dermatitis using topical application of vitamin D3 or of its analog MC903. Methods Mol Biol 1559: 91-106, 2017.

13. Choi J, Kim JR, Kim H, Kim YA, Lee HJ, Kim J and Lee KW: The atopic dermatitis-like symptoms induced by MC903 were alleviated in JNK1 knockout mice. Toxicol Sci 136: 443-449, 2013

14. Liu XJ, Mu ZL, Zhao Y and Zhang JZ: Topical tetracycline improves MC903-induced atopic dermatitis in mice through inhibition of inflammatory cytokines and thymic stromal lymphopoietin expression. Chin Med J (Engl) 129: 1483-1490, 2016.

15. Li M, Hener P, Zhang Z, Kato S, Metzger D and Chambon P. Topical vitamin D3 and low-calcemic analogs induce thymic stromal lymphopoietin in mouse keratinocytes and trigger an atopic dermatitis. Proc Natl Acad Sci USA 103: 11736-11741, 2006.

16. National Research Council (US) Committee for the Update of the Guide for the Care and Use of Laboratory Animals: Guide for the Care and Use of Laboratory Animals. 8th edition. National Academies Press (US), Washington, DC, 2011.

17. Yu J, Luo Y, Zhu Z, Zhou Y, Sun L, Gao J, Sun J, Wang G, Yao X and Li W: A tryptophan metabolite of the skin microbiota attenuates inflammation in patients with atopic dermatitis through the aryl hydrocarbon receptor. J Allergy Clin Immunol 143: 2108-2119.e12,2019.

18. Nomura T and Kabashima K: Advances in atopic dermatitis in 2015. J Allergy Clin Immunol 138: 1548-1555, 2016.

19. Livak KJ and Schmittgen TD: Analysis of relative gene expression data using real-time quantitative PCR and the 2(-Delta Delta C(T)) method. Methods 25: 402-408, 2001.

20. Turner MJ, Dasilva-Arnold SC, Yi Q, Mehrotra P, Kaplan MH and Travers JB: Topical application of a vitamin D analogue exacerbates atopic dermatitis and induces the atopic dermatitis-like phenotype in Stat6VT mice. Pediatr Dermatol 30: 574-578, 2013

21. Irvine AD, McLean WH and Leung DY: Filaggrin mutation associated with skin and allergic diseases. N Engl J Med 365: 1315-1327, 2011

22. Brown SJ and McLean WH: One remarkable molecule: Filaggrin J Invest Dermatol 132 (3 Pt 2): 751-762, 2012.

23. Kelly-Welch AE, Hanson EM, Boothby MR and Keegan AD: Interleukin-4 and interleukin-13 signaling connections maps. Science 300: 1527-1528, 2003

24. Oetjen LK, Mack MR, Feng J, Whelan TM, Niu H, Guo CJ, Chen S, Trier AM, Xu AZ, Tripathi SV, et al: Sensory neurons Co-opt classical immune signaling pathways to mediate chronic itch. Cell 171: 217-228.e13, 2017.

25. Morales JK, Falanga YT, Depcrynski A, Fernando J and Ryan JJ: Mast cell homeostasis and the JAK-STAT pathway. Genes Immun 11: 599-608, 2010.
26. El-Darawish Y, Li W, Yamanishi K, Pencheva M, Oka N, Yamanishi $\mathrm{H}$, Matsuyama $\mathrm{T}$, Tanaka $\mathrm{Y}$, Minato $\mathrm{N}$ and Okamura H: Frontline science: IL-18 primes murine NK cells for proliferation by promoting protein synthesis, survival, and autophagy. J Leukoc Biol 104: 253-264, 2018

27. Alboni S, Montanari C, Benatti C, Sanchez-Alavez M, Rigillo G, Blom JM, Brunello N, Conti B, Pariante MC and Tascedda F: Interleukin 18 activates MAPKs and STAT3 but not NF- $\kappa \mathrm{B}$ in hippocampal HT-22 cells. Brain Behav Immun 40: 85-94, 2014.

28. Stritesky GL, Muthukrishnan R, Sehra S, Goswami R, Pham D, Travers J, Nguyen ET, Levy DE and Kaplan MH: The transcription factor STAT3 is required for T helper 2 cell development. Immunity 34: 39-49, 2011.

29. Lyu Y, Zhu L, Qi R, Xu J, Di Z, Chen H and Gao X: Serum levels of suppressor of cytokine signaling 3 and signal transducer and activator of transcription 3 in childhood atopic dermatitis. Chin Med J (Engl) 127: 2389-2391, 2014.

30. Siegel AM, Stone KD, Cruse G, Lawrence MG, Olivera A, Jung MY, Barber JS, Freeman AF, Holland SM, O'Brien M, et al: Diminished allergic disease in patients with STAT3 mutations reveals a role for STAT3 signaling in mast cell degranulation. J Allergy Clin Immunol 132: 1388-1396, 2013.

31. Theoharides TC, Singh LK, Boucher W, Pang X, Letourneau R, Webster E and Chrousos G: Corticotropin-releasing hormone induces skin mast cell degranulation and increased vascular permeability, a possible explanation for its proinflammatory effects. Endocrinology 139: 403-413, 1998.

32. Crompton R, Clifton VL, Bisits AT, Read MA, Smith R and Wright IM: Corticotropin-releasing hormone causes vasodilation in human skin via mast cell-dependent pathways. J Clin Endocrinol Metab 88: 5427-5432, 2033.

33. Ganceviciene R, Graziene V, Fimmel S and Zouboulis CC: Involvement of the corticotropin-releasing hormone system in the pathogenesis of acne vulgaris. Br J Dermatol 160: 345-352, 2009.

34. Singh LK, Boucher W, Pang X, Letourneau R, Seretakis D, Green $\mathrm{M}$ and Theoharides TC: Potent mast cell degranulation and vascular permeability triggered by urocortin through activation of corticotropin-releasing hormone receptors. J Pharmacol Exp Ther 288: 1349-1356, 1999.

35. Clifton VL, Read MA,LeitchIM,Giles WB, Boura AL, Robinson PJ and Smith R: Corticotropin-releasing hormone-induced vasodilatation in the human fetal-placental circulation: Involvement of the nitric oxide-cyclic guanosine 3',5'-monophosphate-mediated pathway. J Clin Endocrinol Metab 80: 2888-2893, 1995.

36. Vasiadi M,Therianou A, Sideri K, Smyrnioti M, Sismanopoulos N, Delivanis DA, Asadi S, Katsarou-Katsari A, Petrakopoulou T, Theoharides A, et al: Increased serum CRH levels with decreased skin CRHR-1 gene expression in psoriasis and atopic dermatitis. J Allergy Clin Immunol 129: 1410-1413, 2012.

37. Sakai T, Hatano Y, Zhang W, Fujiwara S and Nishiyori R: Knockdown of either filaggrin or loricrin increases the productions of interleukin (IL)-1 $\alpha$, IL-8, IL-18 and granulocyte macrophage colony-stimulating factor in stratified human keratinocytes. J Dermatol Sci 80: 158-160, 2015

38. Holmes J, Fairclough LC and Todd I: Atopic dermatitis and autoimmunity: The occurrence of autoantibodies and their association with disease severity. Arch Dermatol Res 311: 141-162, 2019.

39. Jin JJ, Zou YX and Zeng SW: Risk factors for and expression of immune and inflammatory factors in atopic dermatitis in Chinese population: A birth cohort study. Mol Cell Probes 30: $168-173,2016$.

40. Kabashima-Kubo R, Nakamura M, Sakabe J, Sugita K, Hino R, Mori T, Kobayashi M, Bito T, Kabashima K, Ogasawara K, et al: A group of atopic dermatitis without IgE elevation or barrier impairment shows a high Th1 frequency: Possible immunological state of the intrinsic type. J Dermatol Sci 67: 37-43, 2012.

41. Netea MG, Joosten LA, Lewis E, Jensen DR, Voshol PJ, Kullberg BJ, Tack CJ, van Krieken H, Kim SH, Stalenhoef AF, et al: Deficiency of interleukin-18 in mice leads to hyperphagia, obesity and insulin resistance. Nat Med 12: 650-656, 2006. 